\title{
O gerenciamento do cuidado multidisciplinar no acompanhamento de pacientes portadores de doenças crônicas
}

\section{The management of multidisciplinary care or patients with chronic disease}

\section{Resumo}

O estudo apresenta um modelo de assistência à saúde com base no acompanhamento sistematizado de equipe multidisciplinar a 92 pacientes portadores de doença crônica, com repetidas internações e consultas médicas no período de 12 meses anteriores ao programa, e, em seguida, com a implantação da metodologia por igual tempo, a análise dos resultados obtidos na redução dos custos assistenciais para a operadora do plano de saúde. A investigação foi realizada no período de 01 de janeiro de 2002 a 31 de dezembro de 2002 (sem aplicação do programa) e de 01 de janeiro de 2003 a 31 de dezembro de 2003 (com aplicação do programa).

O trabalho mostra a redução estatisticamente significativa das consultas (média de 15,39 para 9,58), das internações (de 2,11 para 0,85 ), dos custos (de $\mathrm{R} \$ 8.029,32$ para $\mathrm{R} \$ 3.054,03$ ), das internações por motivo de doença principal (54 para 26) e, também, a diminuição das internações devido a complicações da doença (55 para 38). Como conseqüência, uma equação que estabelece um modelo matemático pôde ser desenvolvida, com a finalidade de se obter valor capaz de orçar uma despesa com assistência multidisciplinar daquele paciente, possibilitando um estudo estratégico financeiro para a operadora de plano de saúde, em face à redução dos gastos. O modelo matemático estabelecido é dado pela seguinte equação: $\mathrm{Y}=962+286$ estado civil $-1184 \mathrm{HA}+1070$ internação depois $+84,3$ consulta depois -621 cuidador antes. Via de regra, aplicando a metodologia do programa, alcançamos redução significativa dos custos assistenciais médicohospitalares da operadora do plano de saúde.

\begin{abstract}
This study presents a bealth care program based on systemized multidisciplinary follow-up of 92 patients, carriers of chronic disease, with repeated hospital admissions and medical appointments and analyzes its results in terms of cost reduction for the health plan operator. The investigation covered a period of 12 months before introducing the program (10/01/2002 to 31/12/2002) and the same period after introducing the program (01/01/2003 to 31/12/2003).

The results show a statistically significant reduction of appointments (from a mean of 15,39 to 9,58), hospital admissions (from 2,11

Palavras-chave: Saúde Suplementar; Administração dos Cuidados ao Paciente; Equipe de Assistência ao Paciente; Doença Crônica.

Key Words: Patient Care Management;

Supplemental Health; Patient Care Team;

Chronic Disease.

${ }^{1}$ Enfermeira, Mestre em Ciências da Saúde, Coordenadora do curso de Enfermagem, Faculdade Pitágoras, Federação das UNIMEDS, Minas Gerais, Brasil. ${ }^{2}$ Farmacêutico, Doutor em Epidemiologia, Professor titular, Universidade Federal de Minas Gerais, Belo Horizonte, Minas Gerais, Brasil.
\end{abstract}


to 0,85), costs (from $R \$ 8.029,32$ to $R \$ 3.054,03$ ), as well as a decrease in hospital admissions due to the main disease (from 54 to 26) and to complications (from 55 to 38).

Consequently, an equation establishing a mathematical model could be developed with the purpose of calculating the expenditure with the multidisciplinary care of a patient, allowing the bealth plan operator to conduct a strategic financial analysis for cost reduction. The established mathematical model is represented by the following equation: $Y=962+286$ marital status $-1184 \mathrm{HA}+1070$ admissions after $+84,3$ appointments after -621 caretaker before.

The program improves the health of the patient and results in a significant reduction of the medical and hospital costs for the bealth plan operator.

\section{Introdução}

Analisando o sistema de saúde no Brasil, tanto no âmbito privado quanto no público, pode-se perceber uma carência de planejamento estratégico como instrumento de gestão e assistência no que diz respeito aos cuidados em relação às doenças crônicas ${ }^{1}$. Principalmente no setor privado, especificamente em relação às operadoras de planos de saúde, é fato, ocorre alto custo na assistência médicohospitalar e pouca eficácia no tratamento às doenças crônicas. Conforme apontam alguns autores sobre o assunto, as doenças crônicas necessitam de uma abordagem à saúde mais adaptada às necessidades dos indivíduos ${ }^{1,2,3}$.

Atualmente, fatores como o envelhecimento e a urbanização da população interferem no perfil epidemiológico, refletindo as doenças de grande impacto para os serviços de saúde, e comprometem a qualidade de vida do paciente; são elas: neoplasias, infartos, acidente vascular cerebral (AVC) e pneumonias $2,4,5,6$.

A regulamentação dos planos de saúde, pela Lei 9656/98, que garante amplas coberturas para o tratamento médico-hospitalar, alertou para a necessidade da implementação de novos modelos assistenciais e preventivos de doença como forma de sobrevivência econômico-financeira do sistema de saúde suplementar.

O modelo assistencial de gerenciamento do cuidado multidisciplinar proposto é pautado na integralidade das ações de saúde preventivas de risco, de recuperação e reabilitação do indivíduo, treinando-o para o cuidado com a saúde. Desenvolvido de forma contínua, integrada e humanizada, os resultados do modelo se dão por meio da utilização adequada dos serviços oferecidos ao paciente pela operadora de plano de saúde $e^{1,6,7,8,9}$.

\section{Objetivo}

O estudo visa conhecer o impacto de um modelo de assistência à saúde em pacientes de risco, sobre os custos, internações, número de consultas e complicações, e a partir destes, estabelecer um modelo matemático para que se possa prever os custos e investimentos adequados com base no tamanho e na característica da carteira de clientes.

\section{Metodologia}

O modelo de assistência foi desenvolvido por equipe multidisciplinar-composta por médico, enfermeiro, assistente social, fisioterapeuta e nutricionista -, tendo o enfermeiro assumido a coordenação do monitoramento do paciente durante todo o processo, organizando a atenção, utilizando-se de todos os serviços e instituições que compõem a rede de serviços de saúde e determinando o nível adequado de prestação de serviços, fazendo cumprir o plano de tratamento proposto pelo médico ${ }^{7,8,9}$.

A dinâmica do atendimento se processou por meio da educação para a saúde e treinamento para o paciente e cuidador, em domicílio, no ambulatório e por contato telefônico, e se fundamentou em orientações básicas sobre a doença crônica em questão, sendo este o ponto forte no processo terapêutico.

Gerenciar o cuidado com a saúde do indivíduo por meio contínuo de pontos de atenção à saúde, incluindo $\mathrm{o}$ atendimento a necessidades familiares e sociais foi a base para o modelo aplicado. A gestão do modelo está em garantir o bem-estar, a qualidade de vida e autonomia das pessoas para utilizar de forma adequada os serviços de saúde oferecidos pelo plano de saúde, e, em conseqüência, alcançar a estabilização clínica do indivíduo portador de doença crônica. 
O campo de pesquisa para o estudo foi o plano de saúde da Santa Casa de Belo Horizonte, hospital de grande porte em Minas Gerais. A seleção dos indivíduos foi feita por classificação estabelecida, tendo como base a sua média de gastos médico-hospitalares, sendo recrutados os que obtiveram custo acima de $\mathrm{R} \$ 900,00$, e ainda os que tiveram número de internações acima de dois, e número de consultas acima de 15, em um período de 12 meses.

A amostra foi composta por 92 pacientes, domiciliados em Belo Horizonte (MG), incluídos no programa em dezembro de 2002.

Os dados foram analisados comparando-se o período de 12 meses anterior e posterior ao início do programa. Os softwares estatísticos utilizados foram o SPSS versão $11 \mathrm{e}$ EpiInfo 2002 versão 3.2.2. O teste de normalidade revelou que as variáveis não tinham distribuição normal; por esse motivo foi usado o teste de Wilcoxon.

\section{Resultados}

As principais características da amostra foram: $66,3 \%$ do sexo feminino; a idade média de 71,81 anos; 43,5\% de pacientes casados e $49 \%$, viúvos e solteiros; $93,5 \%$ possuem
A análise descritiva do histórico saúde/doença na amostra estudada revelou: 38 casos de diabetes, 51 casos de hipertensão arterial, 18 casos de DPOC e 21 casos de seqüelas de AVC. Como podemos notar alguns pacientes tinham mais de uma doença.

A amostra demonstra similaridade com os aspectos definidos pela literatura, em face do perfil epidemiológico e social do Brasil, para pacientes portadores de alguma doença crônica ${ }^{1,3,4}$.

No estudo estatístico descritivo, houve redução significativa no valor das variáveis estudadas no período posterior ao da inclusão do paciente no programa, se comparadas ao período anterior (número de internações, consultas, e custo com $\mathrm{p}<0,001$ ), com significância de 0,05 , conforme tabela 1.

No que diz respeito aos motivos das internações, o resultado foi significativamente menor para internações pela doença principal (54 para 26; $\mathrm{p}<0,001$ ) e internações decorrentes de complicações da doença(55 para 38; p0,011). $\mathrm{O}$ mesmo não ocorreu para as internações por motivos secundários, ou seja, intervenções cirúrgicas na sua maioria (13 para 7; p0,153). Os referidos dados demonstram que a

Tabela 1. Média, desvio-padrão, valores mínimos e máximos de consultas, internações e custos antes e depois do programa, em 92 pacientes estudados.

\begin{tabular}{lcccc}
\hline Variável & Média & Desvio-Padrão & Mínimo & Máximo \\
\hline Consultas Antes & 15,39 & 10,10 & 0,00 & 56,00 \\
Consultas Depois & 9,59 & 7,08 & 0,00 & 38,00 \\
Internação Antes & 2,12 & 1,46 & 0,00 & 8,00 \\
Internação Depois & 0,86 & 1,12 & 0,00 & 4,00 \\
Custo total Antes & 8029,33 & 7661,44 & 65,32 & 33022,82 \\
Custo total Depois & 3054,03 & 4747,84 & 14,80 & 30354,30 \\
\hline
\end{tabular}

residência fixa; $90,2 \%$ dos pacientes e $60,9 \%$ dos cuidadores possuem apenas o Ensino Fundamental; $57,1 \%$ dos pacientes possuem médico de referência; $80,4 \%$ possuem cuidador; a renda familiar varia entre 2 e 10 salários mínimos, representando $74,7 \%$ dos pacientes; houve $5,45 \%$ de óbitos durante o programa. assistência prestada ao paciente foi realizada de forma ética e eficaz, não cerceou em nenhum momento o direito do paciente, beneficiário e/ou cidadão de utilizar os recursos oferecidos em cobertura contratual com a empresa contratada por ele para os atendimentos de assistência à saúde ${ }^{3}$. 
Os dados foram então submetidos a uma análise de regressão (regressão linear), com objetivo de se estudar o relacionamento entre uma resposta e covariáveis, uma vez que o modelo de regressão linear é adequado para a modelagem de respostas contínuas. Desta forma, a técnica foi utilizada para a formulação de uma equação matemática que possibilitasse o planejamento de gastos com pacientes portadores de doenças crônicas submetidos à metodologia de assistência estudada. A variável do evento foi custo total depois $=$ Y. Pela análise dos dados, chegou-se à seguinte equação: $\mathrm{Y}=962+286$ Estado civil -1184 Hipertensão arterial +1070 Internação depois $+84,3$ - Consulta depois - 621Cuidador antes. A proposta do modelo matemático apresentado, com base no modelo de regressão linear, prevê os custos gastos com pacientes portadores de doenças crônicas, semelhantes ao da amostra estudada.

As variáveis da equação são significativas no modelo. O coeficiente de determinação (R2) foi de $67,2 \%$ da variabilidade da resposta "custo total depois" entre os indivíduos observados.

O trabalho demonstrou redução nos custos assistenciais para a provedora de saúde. No período anterior ao estudo, houve um gasto de $\mathrm{R} \$ \mathbf{7 3 7 . 8 8 7 , 0 0}$ referente ao custo médico-hospitalar. Após o estudo, os custos referentes a este item caíram para $\mathrm{R} \$ 283.276,00$. Considerando que foi acrescido um custo operacional de $\mathrm{R} \$ 143.074,00$, o custo total depois do estudo ficou em $\mathrm{R} \$ 426.350,00$. Deste modo, comparando-se o custo antes do estudo e o após, verifica-se que houve uma redução de $\mathrm{R} \$ 311.537,00$, representando $42,23 \%$ de economia para o plano de saúde.

\section{Conclusão}

$\mathrm{Na}$ amostra estudada, houve preponderância de indivíduos: hipertensos, do sexo feminino e com grau de escolaridade (pacientes e cuidadores) representado pelo Ensino Fundamental. Isso retrata as últimas pesquisas apresentadas pelo $\mathrm{IBGE}^{5}$ e confirma o perfil epidemiológico da população na atualidade conforme a literatura ${ }^{1,4,6}$.

Neste estudo, a redução de $66,7 \%$ do número de consultas $(15,39$ para 9,58$)$ levou a uma redução de $37,5 \%$ no custo, apenas com as despesas médico-hospitalares. Sabendo-se que, de forma geral, as consultas médicas desencadeiam o processo terapêutico, internações e pedidos de exames para diagnóstico e tratamento, pode-se admitir que os resultados obtidos foram vantajosos para os pacientes portadores de doenças crônicas, isto é, houve melhora da eficácia em relação ao modelo convencional de atenção à saúde para o tratamento de pacientes.

Diante do exposto e por meio dos resultados obtidos no estudo, ficou comprovado que o presente modelo diferenciado em assistência à saúde, diverso do que habitualmente é oferecido à população, apresenta resultados positivos para a provedora de saúde na redução dos custos e, para os pacientes, sugere estabilização da doença.

\section{Referências}

1. Starfield B. Atenção primária-equilíbrio entre necessidades de saúde, serviços e tecnologias. Brasilia (DF): UNESCO, Ministério da saúde; 2002. 726p.

2. Mendes EV. Os sistemas de serviços de saúde: o que os gestores deveriam saber sobre essas organizações complexas. Fortaleza (CE): Escola de Saúde Pública do Ceará; 2002. 3. Cecílio LC. Inventando a mudança na saúde. São Paulo: Hucitec; 1984.

4. Chaimowicz F. Os idosos brasileiros no século XXI demografia, saúde e sociedade. Belo Horizonte (MG): Postgraute Brasil; 1998.

5. IBGE. Pesquisa nacional por amostra de domicílios 2003; Brasil em síntese 2003; Síntese de indicadores sociais 2003; Pesquisa sobre acesso a serviços de saúde 2003. Disponível em: http://www.ibge.gov.br. Acesso em: abr., dez. 2004; maio 2005.

6. Duarte Y, Diogo MJD. Atendimento domiciliar um enfoque gerontológico. São Paulo: Atheneu; 2000.

7. Ciampone MHT. Metodologia do planejamento aplicada à enfermagem. In: Kurcgant P.(org.). Administração em enfermagem. São Paulo: [s.n]; 1991.

8. Horta WA. Processo de enfermagem. São Paulo: EPU; 1979.

9. Wright LM. Enfermeiras e famílias: um guia para 
avaliação e intervenção na família. 3 ed. São Paulo: Roca;

2002.

\section{Endereço para correspondência:}

Rua Luiz Castanhedes, 578

Belo Horizonte (MG)

CEP: 31155-140

\section{Endereço eletrônico:}

regina@unimedmg.com.br 